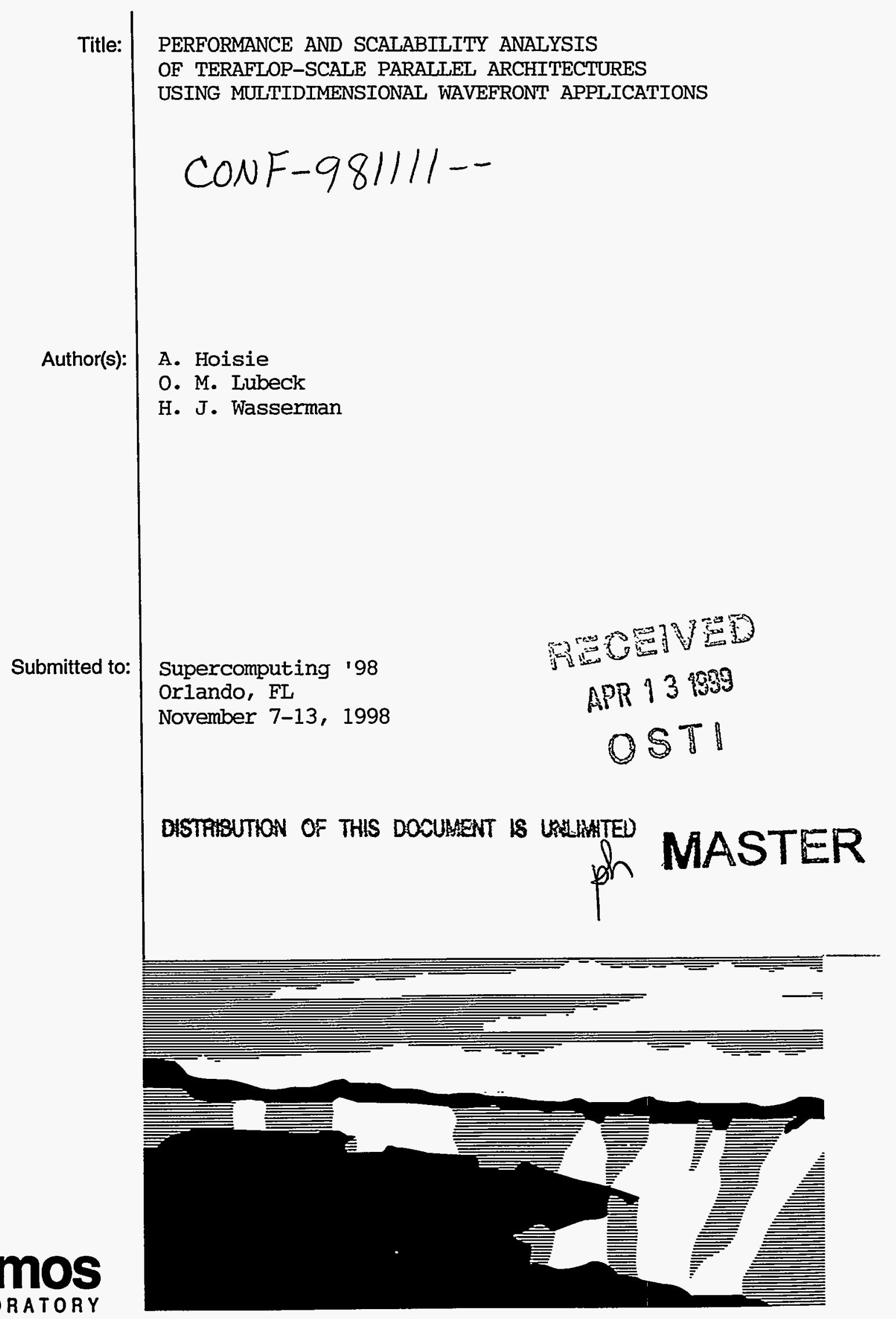

Los Alamos National Laboratory, an affimative actionequal opportunity employer, is operated by the University of Califomia for the U.S. Department of Energy under contract W-7405-ENG-36. By acceptance of this article, the publisher recognizes that the U.S. Govemment retains a nonexclusive, royalty-free license to publish or reproduce the published form of this contribution, or to allow others to do so, for U.S. Govemment purposes. Los Alamos National Laboratory requests that the publisher identify this article as work performed under the auspices of the U.S. Department of Energy. The Los Alamos National Laboratory strongly supports academic freedom and a researcher's right to publish; as an institution, however, the Laboratory does not endorse the viewpoint of a publication or guaranteo its technical correctness. 


\section{DISCLAIMER}

This report was prepared as an account of work sponsored by an agency of the United States Government Neither the United Strtes Government nor any agency thereof, nor any of their employees, makes any warranty, express of implied, or essumes any legal liability or responsibility for the aceuracy, completeress, or usefulness of any information, apparatus, product, of process diselosed, or represents that its use would not infringe privately owned rights. Reference berein to any specific commercial produch, process, or service by trade name, trademart, imanufece. turer, or otherwise does aot necessarily constiture or imply its eadorsement, recom. mendation, or favoring by the United States Government or any agency thereof. The views and opinions of anthors expresed herein do not necessarily sate or reflect those of the United States Government or any agency thereof. 


\section{DISCLAIMER}

Portions of this document may be illegible in electronic image products. Images are produced from the best available original document. 


\title{
Performance and Scalability Analysis of Teraflop-Scale Parallel Architectures Using Multidimensional Wavefront Applications
}

\author{
Adolfy Hoisie, Olaf Lubeck, and \\ Harvey Wasserman \\ $<$ hoisie, oml, hjw> @lanl.gov \\ Scientific Computing Group \\ Los Alamos National Laboratory \\ Los Alamos, NM 87545
}

\begin{abstract}
We develop a model for the parallel performance of algorithms that consist of concurrent, twodimensional wavefronts implemented in a message passing environment. The model, based on a LogGP machine parametrization, combines the separate contributions of computation and communication wavefronts. We validate the model on three important supercomputer systems, on up to 500 processors. We use data from a deterministic particle transport application taken from the ASCI workload, although the model is general to any wavefront algorithm implemented on a 2-D processor domain. We also use the validated model to make estimates of performance and scalability of wavefront algorithms on 100-TFLOPS computer systems expected to be in existence within the next decade as part of the ASCI program and elsewhere. In this context, we analyze two problem sizes. Our model shows that on the largest such problem (1 billion cells), inter-processor communication performance is not the bottleneck. Single-node efficiency is the dominant factor.
\end{abstract}

\section{Introduction}

Wavefront techniques are used to enable parallelism in algorithms that have recurrences by breaking the computation into segments and pipelining the segments through multiple processors [1]. First described as "hyperplane" methods by Lamport [2], wavefront methods now find application in several important areas including particle physics simulations [3], parallel iterative solvers [4], and parallel solution of triangular systems of linear equations [5-7].

Wavefront computations present interesting implementation and performance modeling challenges on distributed memory machines because they exhibit a subtle balance between processor utilization and communication cost. Optimal task granularity is a function of machine parameters such as raw computational speed, and interprocessor communication latency and bandwidth. Although it is simple to model the computation-only portion of a single wavefront, it is considerably more complicated to model multiple wavefronts existing simultaneously, due to potential overlap of computation and communication and/or overlap of different communication or computation operations individually. Moreover, specific message passing synchronization methods impose constraints that can further limit the available parallelism in the algorithm. A realistic scalability analysis must take into consideration these constraints.

Much of the previous parallel performance modeling of software-pipelined applications has involved algorithms with one-dimensional recurrences and/or one-dimensional processor decompositions [5-7]. A key contribution of this paper is the development of an analytic performance model of wavefront algorithms that have recurrences in multiple dimensions and that have been partitioned and pipelined on multidimensional processor grids. We use a "compact application" called SWEEP3D, a timeindependent, Cartesian-grid, single-group, "discrete ordinates" deterministic particle transport code taken from the DOE Accelerated Strategic Computing Initiative (ASCI) workload. Estimates are that deterministic particle transport accounts for $50-80 \%$ of the execution time of many realistic simulations on current DOE systems; this percentage may expand on future 100-TFLOPS systems. Thus, an equally-important contribution of this work is the use of our model to explore SWEEP3D scalability and to show the sensitivity of SWEEP3D to per-processor sustained speed, and MPI latency and bandwidth on futuregeneration systems.

Efforts devoted to improving performance of discrete ordinates particle transport codes date back many years and have extended recently to massively-parallel systems [8-12]. Research has included models of performance as a function of problem and machine size, as well as other characteristics of both the simulation and the computer system under study. For example, Koch, Baker, and Alcouffe [3] developed a parallel efficiency formula that considered computation only, while Baker and Alcouffe [9] developed a model specific to CRAY T3D put/get communication. However, these previous models had limiting assumptions about the computation and/or the target machines.

In this work, we model parallel discrete ordinates 
transport and account for both computation and communication. We validate the model on several architectures within the realistic limits of all parameters appearing in the model. Sections 2 and 3 of the paper briefly describe the algorithm and its implementation. Sections 4 and 5 derive the performance model and give validation results. In the final sections of the paper, the model is used to estimate SWEEP3D performance on future generation parallel systems, showing the sensitivity of this application to system computation and communication parameters.

Note that although we present results for three different parallel systems, no comparison of achieved system performance or scalability is intended. Rather, measurements from the three systems are presented in an effort to demonstrate generality of the performance model and sensitivity of application performance to machine parameters.

\section{Description of Discrete Ordinates Transport}

Although much more complete treatments of discrete ordinates neutron transport have appeared elsewhere [1215], we include a brief explanation here to make clear the origin of the wavefront process in SWEEP3D. The basis for neutron transport simulation is the time-independent, multigroup, inhomogeneous Boltzmann transport equation, which is formulated as

$$
\begin{aligned}
& \nabla \cdot \Omega \Psi(\mathbf{r}, \mathrm{E}, \Omega)+\iint \sigma(\mathbf{r}, \mathrm{E}) \Psi(\mathbf{r}, \mathrm{E}, \Omega)= \\
& \iint \mathrm{dE}^{\prime} \mathrm{d}^{\prime}\left(\mathbf{r}, \mathrm{E}^{\prime} \rightarrow \mathrm{E}, \Omega \cdot \Omega^{\prime}\right) \Psi\left(\mathrm{r}, \mathrm{E}^{\prime}, \Omega^{\prime}\right)+ \\
& (1 / 4 \pi) \iint \mathrm{d} \mathrm{E}^{\prime} \mathrm{d} \Omega^{\prime} \chi\left(\mathrm{r}, \mathrm{E}^{\prime} \rightarrow \mathrm{E}\right) \vee \sigma\left(\mathbf{r}, \mathrm{E}^{\prime}\right) \Psi\left(\mathbf{r}, \mathrm{E}^{\prime}, \Omega^{\prime}\right)+ \\
& \mathrm{Q}(\mathbf{r}, \mathrm{E}, \Omega) .
\end{aligned}
$$

The unknown quantity is $\Psi$, which represents the flux of particles at the spatial point $\mathbf{r}$ with energy $E$ traveling in direction $\Omega$.

Numerical solution involves complete discretization of the multi-dimensional phase space defined by $r, \Omega$, and $E$. Discretization of energy uses a "multigroup" treatment, in which the energy domain is partitioned into subintervals in which the dependence on energy is known. In the discrete ordinates approximation, the angular-direction $\Omega$ is discretized into a set a quadrature points. This is also referred to as the $S_{N}$ method, where (in 1D) $N$ represents the number of angular ordinates used. The discretization is completed by differencing the spatial domain of the problem on to a grid of cells.

The numerical solution to the transport equation involves an iterative procedure called a "source iteration" (see Ref. 13). The most time-consuming portion is the "source correction scheme," which involves a transport sweep through the entire grid-angle space in the direction of particle travel. A lower triangular matrix is obtained, as such one needs to go through the grid only once in inverting the iteration matrix. In Cartesian geometries, each octant of angles has a different sweep direction through the mesh, and all angles in a given octant sweep the same way.

For a given discrete angle, each grid cell has a spatially-exact particle "balance equation" with seven unknowns. The unknowns are the particle fluxes on the six cell faces and the flux within the cell. Boundary conditions and the spatial differencing approximation are used to provide closure to the system. Boundary conditions (typically vacuum or reflective) allow the sweep to be initiated at the object's exterior. Thereafter, for any given cell, the fluxes on the three incoming cell planes for particles traveling in a given discrete angle are known and are used to solve for the cell center and the three cell faces through which particles leave the cell. Thus, each interior cell requires in advance the solution of its three upstream neighboring cells $-\mathrm{a}$ three-dimensional recursion. This is illustrated in Figure 1 for a 1-D arrangement of cells and in Figure 2 for a 2-D grid.

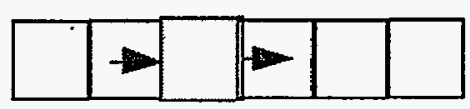

Figure 1. Dependences for a 1-D Transport Sweep.

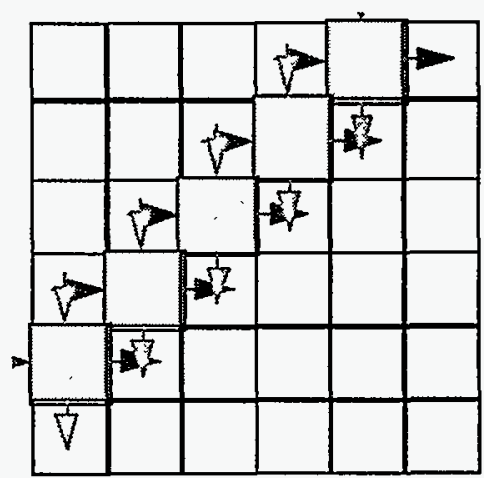

Figure 2. 2-D Transport Sweep along a Diagonal Wavefront.

\section{Parallelism in Discrete Ordinates Transport}

The only inherent parallelism is related to the discretization over angles. However, reflective boundary conditions limit this parallelism to, at most, angles within a single octant.

The two-dimensional recurrence may be partially eliminated because solutions for cells within a diagonal are independent of each other (as shown in Figure 2). The success of this "diagonal sweep" scheme on SIMD computers such as single-processor vector systems (using 2-D plane diagonals) and the Thinking Machines, Inc. Connection Machine (using 3-D body diagonals) has been demonstrated [3]. 
Diagonal concurrency can also be the basis for implementation of a transport sweep using a decomposition of the mesh into subdomains using message passing to communicate the boundaries between processors, as described in [12] and shown in Figure 3. The transport sweep is performed subdomain by subdomain in a given angular direction. Each processor's exterior surfaces are computed by, and received in a message from, "upstream" processors owning the subdomains sharing these surfaces.

However, as pointed out by Baker [9] and Koch [3], the dimensionality of the $S_{N}$ parallelism is always one order lower than the spatial dimensionality because recursion in one spatial direction cannot be eliminated.

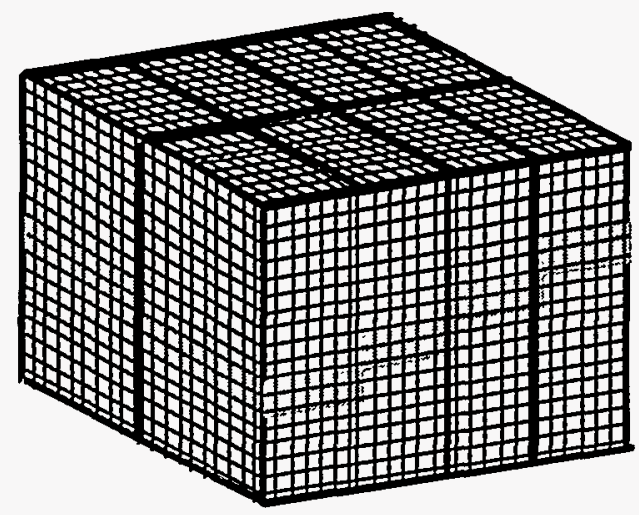

Figure 3. Illustration of the 2-D Domain decomposition on eight processors with 2 k-planes per block. The transport sweep has started at top of the processor in the foreground. Concurrently-computed cells are shaded.

Because of this, parallelization of the 3-D $S_{N}$ transport in SWEEP3D uses a 2-D processor decomposition of the spatial domain.

Parallel efficiency would be limited if each processor computed its entire local domain before communicating information to its neighbors. A strategy in which blocks of planes in one direction ( $k$, in the current implementation) and angles are pipelined through this 2-D processor array improves the efficiency, as shown in Figure 3. Varying the $\mathrm{k}$ - and angle-block sizes changes the balance between parallel utilization and communication time.

\section{A Performance Model for Parallel Wavefronts}

This section describes a performance model of a message passing implementation of SWEEP3D. Our model uses a pipelined wavefront as the basic abstraction and predicts the execution time of the transport sweep as a function of primary computation and communication parameters. We use a two-parameter (latency/bandwidth) linear model for communication performance, which is equivalent to the LogGP model [16]. We use the term latency to mean the sum of $L$ and $o$ in the LogGP framework, and bandwidth to mean the inverse of G. Since different implementations of MPI use different buffering strategies as a function of message size, a single set of latency/bandwidth parameters describes a limited range of message sizes. Consequently, multiple sets are used to describe the entire range. Computation time is parameterized by problem size, the number of floating-point calculations per grid point, and a characteristic singleCPU floating-point speed.

\subsection{Pipelined Wavefront Abstraction}

An abstraction of the SWEEP3D algorithm partitioned for message passing on a 2-D processor domain (ij plane) is described in Figure 4. The inner-loop body of this algorithm describes a wavefront calculation with recurrences in two dimensions. Each processor must wait for boundary information from neighboring processors to the north and west before computing on its subdomain. For convenience, we assume that the implementation uses MPI with synchronous, blocking sends/receives. There is little loss of generality in this assumption since the subdomain computation must wait for message receipt. Multiple waves initiated by the octant, angle-block and k- block loops are pipelined one after another as shown in Figure 5, in which two inner loop bodies (or "sweeps") are executing on a $P x$ by $P y$ processor grid. Each diagonal line of processors is executing the same k-block loop iteration in parallel on a different subdomain; two such diagonals are highlighted in the figure.

Using this pipeline abstraction as the foundation, we can build a model of execution time for the transport sweep. The number of steps required to execute a computation of $N_{\text {sweep }}$ wavefronts, each with a pipeline length of $N_{s}$ stages and a repetition delay of $d$ is given by equation (1).

$$
\text { Steps }=N_{\mathrm{s}}+d\left(N_{\text {sweep }}-1\right) \text {, }
$$

The first wavefront exits the pipeline after $N_{s}$ stages and subsequent waves exit at the rate of $1 / d$.

The pipeline consists of both computation and communication stages. The number of stages of each kind and the repetition delay per wavefront need to be determined as a function of the number of processors and shape of the processor grid. The cost of each individual computation/communication stage is dependent on problem size, processor speed and communication parameters.

\subsection{Computation Stages}

Figure 5 shows that the number of computation stages is simply the number of diagonals in the grid. A 


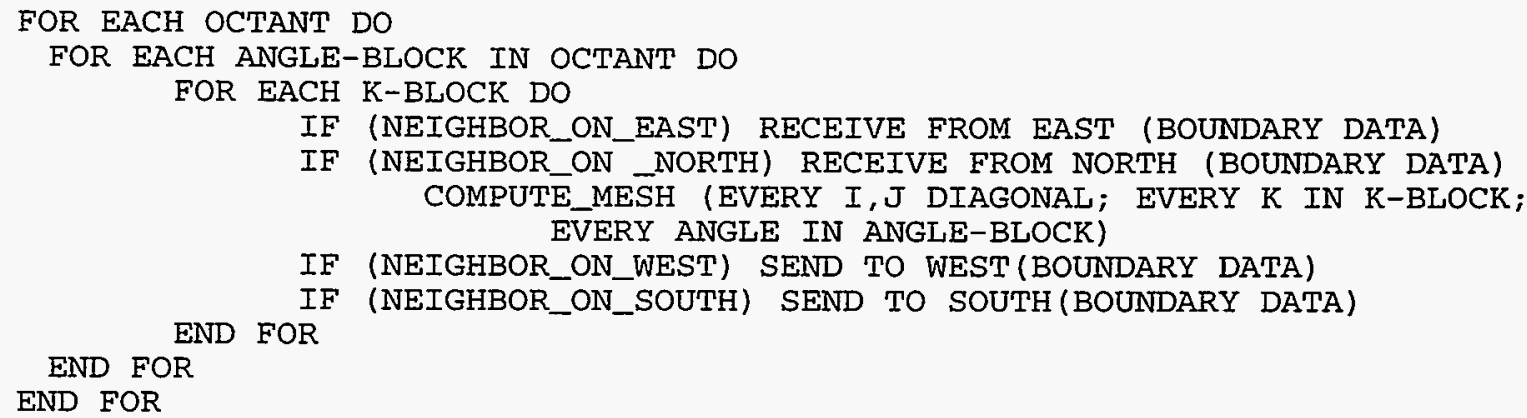

Figure 4. Pseudo Code for the wavefront Algorithm

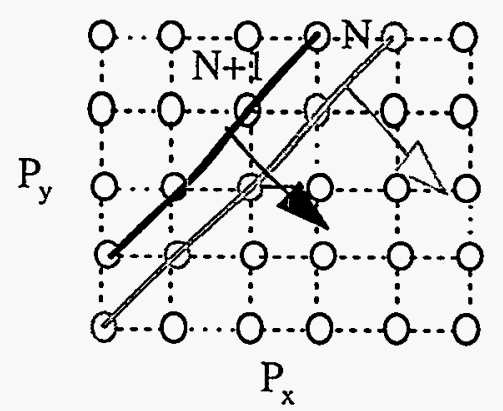

Figure 5. Multidimensional Pipelined Wavefronts

different number of processors is employed at each stage but all stages take the same amount of time since processors on a diagonal are executing concurrently. The cost of one computational stage is thus the time to complete one COMPUTE_MESH function (see algorithm abstraction above) on a processor's subdomain. The discussion can be summarized with two equations. Equation (2) gives the number of computation steps in the pipeline,

$$
N_{s}^{\text {comp }}=P_{x}+P_{y}-1
$$

and Equation 3 gives the cost of each step,

$T_{c p u}=\left(\frac{N_{x}}{P_{x}}+\frac{N_{y}}{P_{y}}+\frac{N_{z}}{K_{b}}+\frac{N_{a}}{A_{b}}\right) \frac{N_{\text {flops }}}{R_{\text {flops }}}$

where $N_{x}, N_{y}$, and $N_{z}$ are the number of grid points in each direction; $K_{b}$ is the size of the k-plane block; $A_{b}$ is the size of the angular block; $N_{\text {flops }}$ is the number of floating-point operations per gridpoint; and $R_{\text {flops }}$ is a characteristic floating-point rate for the processor. The next sweep can begin as soon as the first processor completes its computation so the repetition delay, $d^{\text {comp }}$, is 1 computational step (i.e., the time for completing one diagonal in the sweep).

\subsection{Communication Stages}

The number and cost of communication stages are dependent on specific characteristics of the communication system. The effect of blocking synchronous communications is that messages initiated by the same processor occur sequentially in time and messages must be received in the same order that they are sent. As implemented, the order of receives is first from the west, then from the north, and the order of sends is first to the east and then to the south. These rules lead to the ordering (and concurrency) of the communications for a $4 \times 4$ processor grid as shown in Figure 6 for a sweep that starts in the upper-left quadrant.

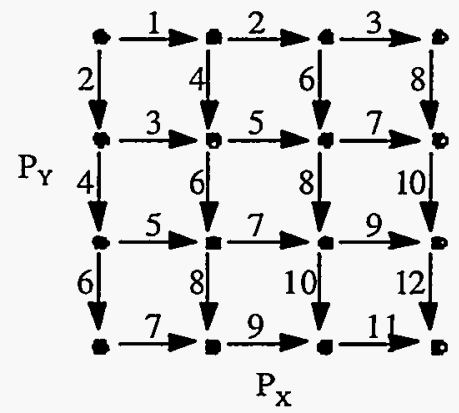

Figure 6 Communication Pipeline.

In Figure 6 edges labeled with the same number are executed simultaneously and the graph shows that it takes 12 steps to complete one communication sweep on a $4 \times 4$ processor grid. We assume that a logical processor mesh can be imbedded into the machine topology such that each mesh node maps to a unique processor and each mesh edge maps to a unique router link. One can generalize the number of stages to a grid of $P_{x}$ by $P_{y}$ processors by observing that communication for each row of processors is initiated by a message from a north neighbor in the first column of processors. South-going messages in the first column of processors occur on every other step since each processor in the column a) has no west neighbor, and b) must send east before sending south. Thus the last processor in the first column receives a message on step $2\left(P_{y^{-}}\right.$ $1)$. This initiates a string of west-going messages along the last row that are also sent on every other step, and the number of stages in the communication pipeline is given by 


$$
N_{s}^{c a r m}=2\left(P_{y}-1\right)+2\left(P_{x}-1\right)
$$

Analogous to the computational pipeline, different stages of the communication pipeline have different numbers of point-to-point communications. However, since these occur simultaneously, the cost of any single communication stage is the time of a one-way, nearest neighbor communication. This time is given by:

$$
T_{m s g}=t_{0}+\frac{N_{m s g}}{B}
$$

where latency + overhead $\left(t_{0}\right)$ and bandwidth $(B)$, are defined in LogGP as noted above.

The repetition delay for the communication pipeline, $d^{\text {romm }}$, is 4 because a message sent from the top-left processor (processor 0) to its east neighbor (processor 1) on the second sweep cannot be initiated until processor 1 completes its communication with its south neighbor from the first sweep (Figure 5).

\subsection{Combining Computation and Communication Stages}

In the previous two sections, we derived formulas for the modeling of SWEEP3D that are general for any pipelined wavefront computation. We can summarize the discussion in two equations that give the separate contributions of computation and communication:

$$
\begin{aligned}
& T^{\text {comp }}=\left[\left(P_{x}+P_{y}-1\right)+\left(N_{\text {sweep }}-1\right)\right] * T_{c p u} \\
& T^{\text {comm }}=\left[2\left(P_{x}+P_{y}-2\right)+4\left(N_{\text {sweep }}-1\right)\right]^{*} T_{m s g}
\end{aligned}
$$

The major remaining question is whether the separate contributions, $T^{\text {comp }}$ and $T^{\text {comm }}$, can be summed to derive the total time. They would not be additive if there were any additional overlap of communication with computation not already accounted for in each term. To see that this is not the case, consider the task graph for an execution consisting of two wavefronts on a $3 \times 3$ processor grid (Figure 7). This graph shows communication tasks (circles numbered with a send/receive processor pair) and computation tasks (squares numbered by a computing processor). The total number of stages in the combined communication/computation pipeline is equal to the number of nodes (of each type) in the longest path through the graph (the critical path) shown in red in the

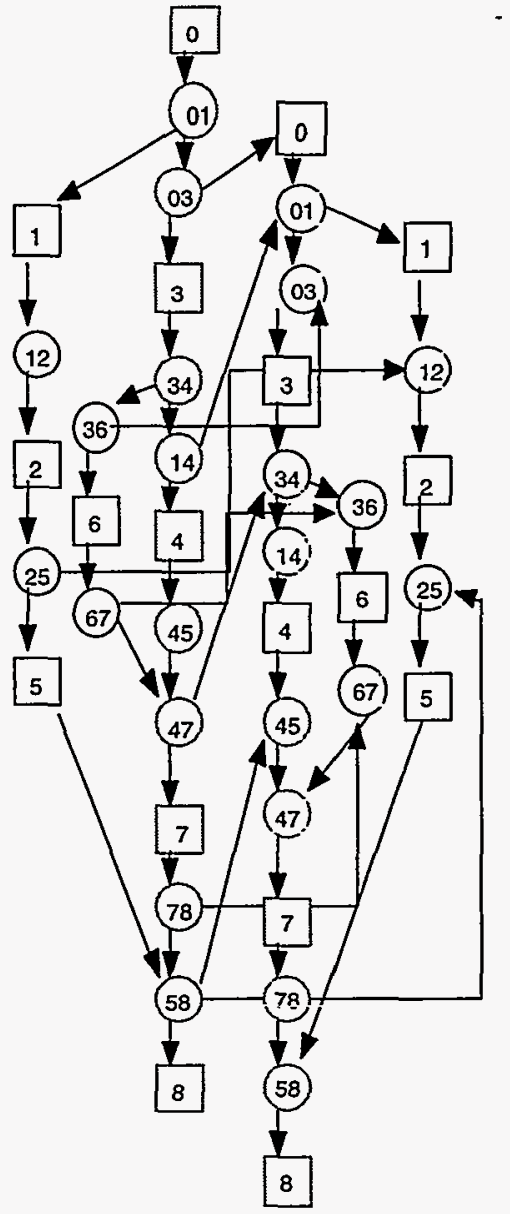

\section{Figure 7. Pipelined Wavefront Task Graph.}

figure. The critical path for the first sweep can be counted from Figure 7: 5 computational tasks and 8 communication tasks. This result is exactly the number given by eqns. (2) and (4). One can further verify that there is no further overlap between two pipelined sweeps other than the predicted sum of eqns. (6) and (7). The second sweep completes exactly 1 computation and 4 communication steps after the first.

In summary, total time for the sweep algorithm is the sum of eqns. (6) and (7), where Tcpu is given by eqn. (3) and Tmsg is given by eqn. (5). The validation of the model against experiment involves the measurement and/or modeling of Tmsg and Tcpu. We take Tmsg to be the time needed for the completion of a send/receive pair of an appropriate size and Tcpu to be the computational work associated with the subgrid computation on each processor.

\section{Validation of the Model}

In this section, we present results that validate the model with performance data from SWEEP3D on three different machines, with up to 500 processors, over the entire range of the various model parameters. Inspection 
of eqns. (6) and (7) leads to identification of the following validation regimes:

$N_{\text {sweep }}=1$ : This case validates the number of pipeline stages in $T^{\text {comp }}$ and $T^{\text {comm }}$, as functions of $\left(P_{x}+P_{y}\right)$, in the available range of processor configurations.

$N_{\text {sweep }} \sim\left(P_{x}+P_{y}\right)$ : Validation of a case where the contributions of the $\left(P_{x}+P_{y}\right)$ and $N_{\text {sweep }}$ terms are comparable.

$N_{\text {sweep }} \gg\left(P_{x}+P_{y}\right):$ This case validates the repetition rate of the pipeline.

For each of these three cases, we analyze problem sizes chosen in such a way as to make:

$T^{\text {comp }} \gg T^{\text {comm }}$; (validate eqn. (6) only)

$T^{\text {comp }}=0$; (validate eqn. (7) only)

$T^{\text {comp }} \sim T^{\text {comm }}$ ( (validate the sum of eqns. (6) and (7)).

\section{$5.1 \quad N_{\text {sweep }}=1$}

For a single sweep, the coefficients of $T_{m s g}$ and $T_{c p u}$ in equations 6 and 7 represent the number of communication and computation stages in the pipeline, respectively. Any overlap in communication or computation during the single sweep of the mesh is encapsulated in the respective coefficients. In hypothetical problems with $T_{m s g}-T_{c p u}$, and in the limit of large processor configurations (large $P_{x}+P_{y}$ ), equations 6 and 7 show that the communication component of the elapsed time would be twice as large as the contribution of the computation time. In reality, for problem sizes and partitionings reasonably designed (small subgrid surface-to-volume ratio), $T_{c p u}$ is considerably larger than $T_{m s g}$. Computation is the dominant component of the elapsed time.

This is apparent in Figure 8, which presents the modelexperiment comparison for a weak scalability analysis of a $16 \times 16 \times 1000$ subgrid size sweeping only one octant. This size was chosen to reflect an estimate of the subgrid size for a 1-billion cell-problem running on a machine with about 4,000 processors; the former is a canonical goal of ASCI and the latter is simply an estimate of the machine size that might satisfy a 3-TFLOPS peak performance requirement. In a "weak scalability" analysis, the problem size scales with the processor configuration so that the computational load per processor stays constant. This experiment shows that the contribution of communication is small (in fact, the model shows that it is about 150 times smaller than computation), and the model is in very good agreement with the experiment.

In the absence of communication our model reduces to the linear "parallel computational efficiency" models used by Baker [9] and Koch [3] for $S_{N}$ performance, in which parallel computational efficiency is defined as the fraction of time a processor is doing useful work.

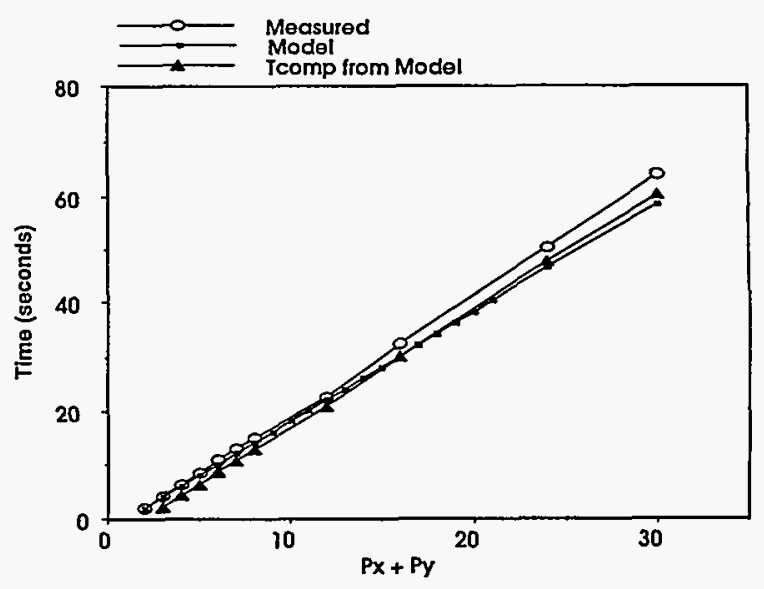

Figure 8. $T^{\text {comp }}$ dominant. $N_{\text {sweep }}=1$. IBM RS/6000.

To validate the case with $N_{\text {sweep }}=1$ and "comparable" contributions of communication and computation we had to use a subgrid size that is probably unrealistic for actual production simulation purposes $(5 \times 5 \times 1)$. Even with this size computation outweighs communication by about a factor of 6 . Figure 9 depicts a weak scalability analysis on the SGI Origin 2000 for this size. The modelexperiment agreement is again very good.

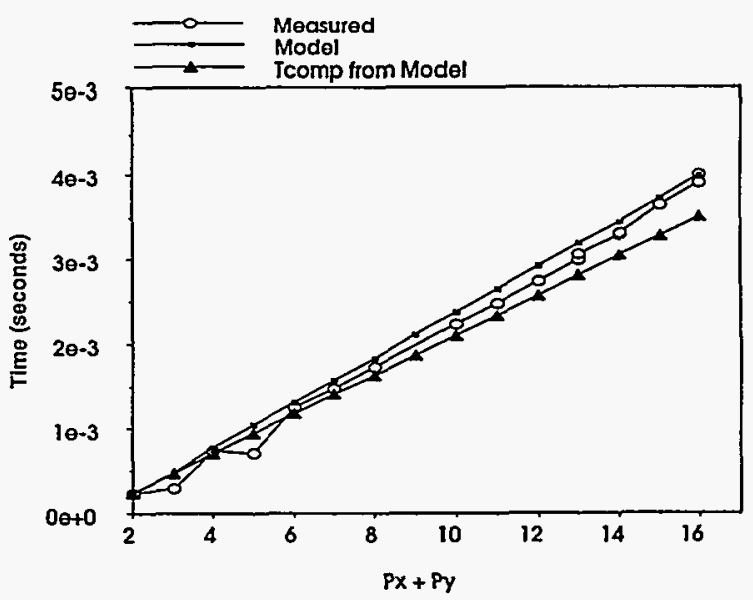

Figure 9. $T^{\text {somp }} \sim T^{\text {comm }} . N_{\text {sweep }}=1$. SGI Origin.

Validation of cases where $T^{\text {comp }}=0$ involved the development of a new code to simulate the communication pattern in SWEEP3D in the absence of computation. The code developed for this purpose simply implements a receive-west, receive-north, send-south, send-east communication pattern enclosed in loops that initiate multiple waves. Figure 10 shows a very good agreement of the model with the measured data from this code.

\section{$5.2 N_{\text {sweep }} \sim\left(P_{x}+P_{y}\right)$}

As described in Section 4, sweeps of the domain generated by successive octants, angle blocks, and $\mathrm{k}$ - 


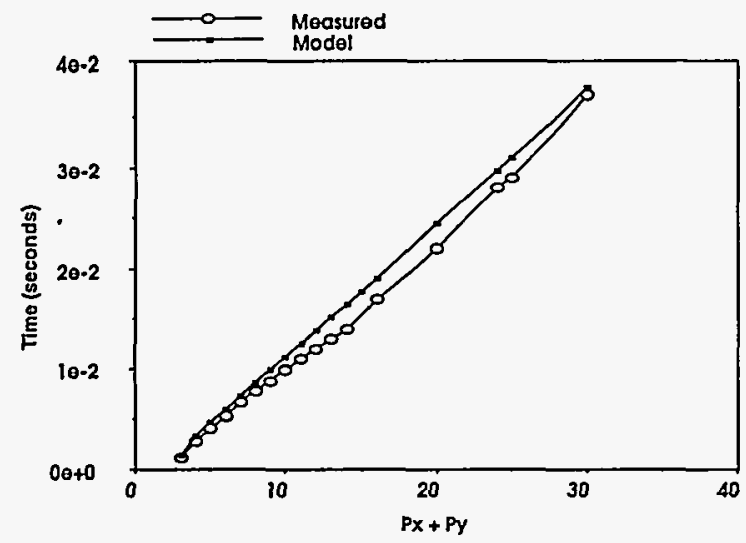

Figure 10. $T^{\text {somp }}=0 . N_{\text {sweep }}=1$. SGI Origin.

plane blocks are pipelined, with the depth of the pipeline, $N_{\text {sweep }}$, given by the product of the number of octants, angle blocks, and k-plane blocks. We can select k-plane and angle block sizes so that $N_{\text {sweep }}=10$, which, in turn, balances the contribution of $N_{\text {sweep }}$ and $\left(P_{x}+P_{y}\right)$ for processor configurations used in this work. In Figure 11 the comparison using a data size for which $T^{\text {comp }}$ is dominant is presented, showing an excellent agreement with the measured elapsed time.

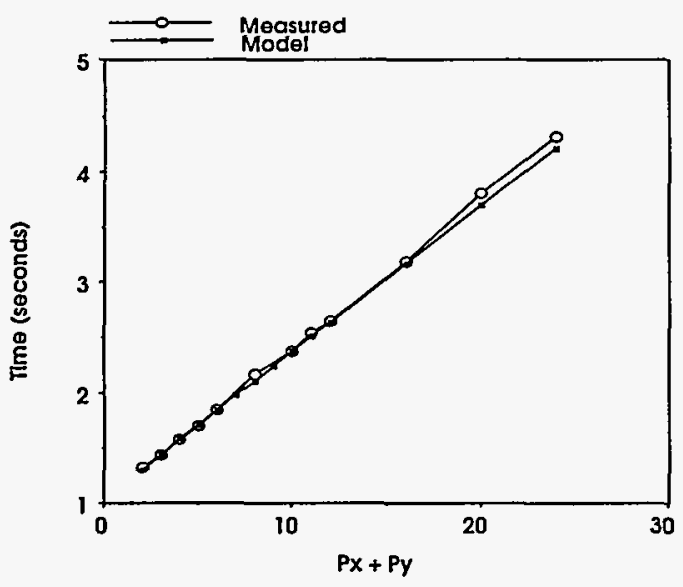

Figure 11. $T^{\text {somp }}$ dominant. $N_{\text {sucep }}=10$, SGI Origin

The case with no computation is in fact a succession of 10 sweeps of the domain, with the communication overlap described by equation 6 . Figure 12 shows a very good agreement with experimental data for this case.

An excellent model-experiment agreement is similarly shown in Figure 13, for a subgrid size $5 \times 5 \times 1$, which leads to balanced contributions of the communication and computation terms to the total elapsed time of SWEEP3D.

\subsection{Nweep $\gg P x+P y$}

We present model-data comparisons using weak scalability experiments for cases in which $N_{\text {sweep }}$ is large

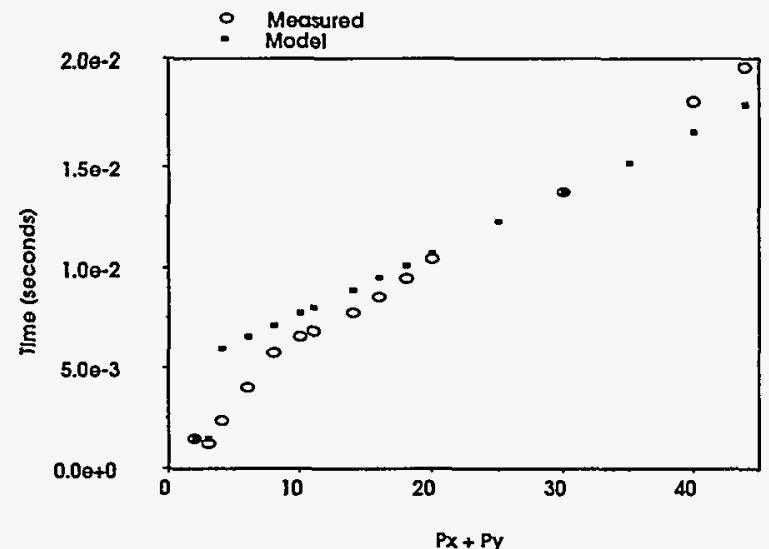

Figure 12. $T^{\text {comp }}=0 . N_{\text {seeep }}=10$. CRAY T3E.

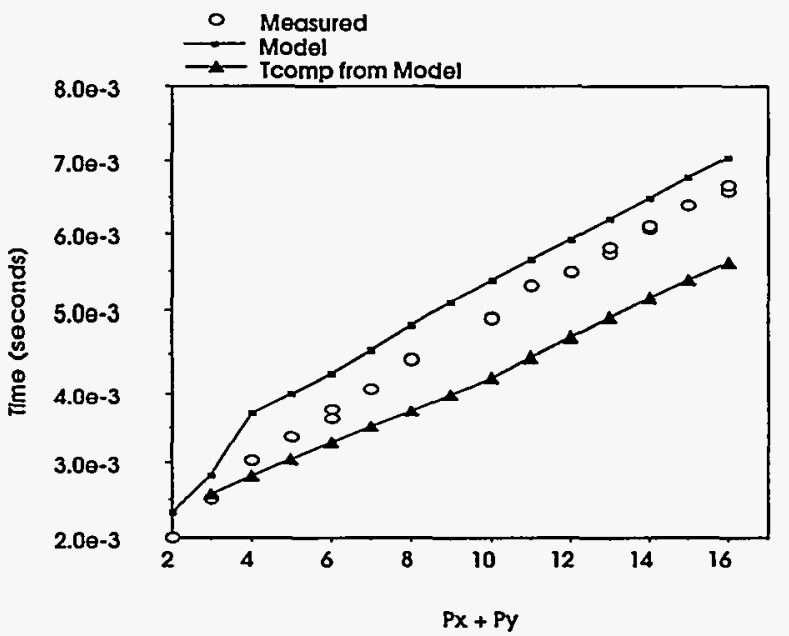

Figure $13 T^{\text {somp }}$ dominant. $N_{\text {ssecep }}=10$. SGI Origin

compared with $(P x+P y)$ in Figure $14(6 \times 6 \times 360$ subgrid; $\left.T^{\text {comp }} \sim T^{\text {comm }}\right)$ and in Figure $15(16 \times 16 \times 1000$ subgrid; $T^{\text {comp }}$ dominant). The model is in good agreement with the measured execution times of SWEEP3D in both cases.

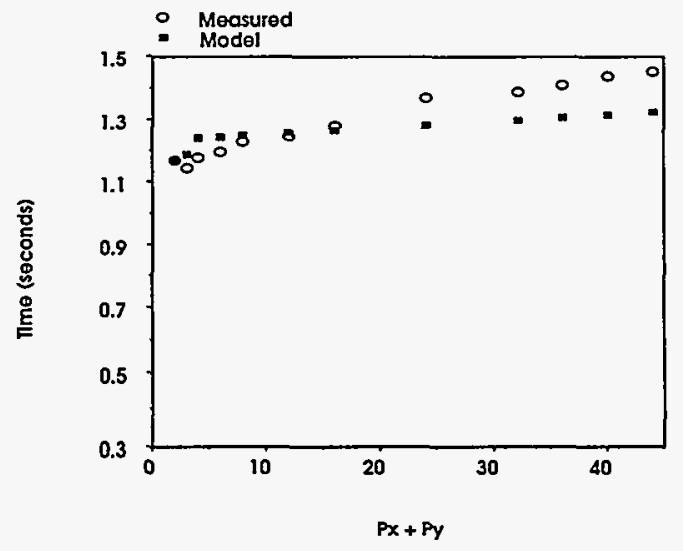

Figure 14. $T^{\text {comp }} \sim T^{\text {comm }} .6 \times 6 \times 360 . N_{\text {sweep }}$ large. CRAY T3E. $K_{b}=10$. 


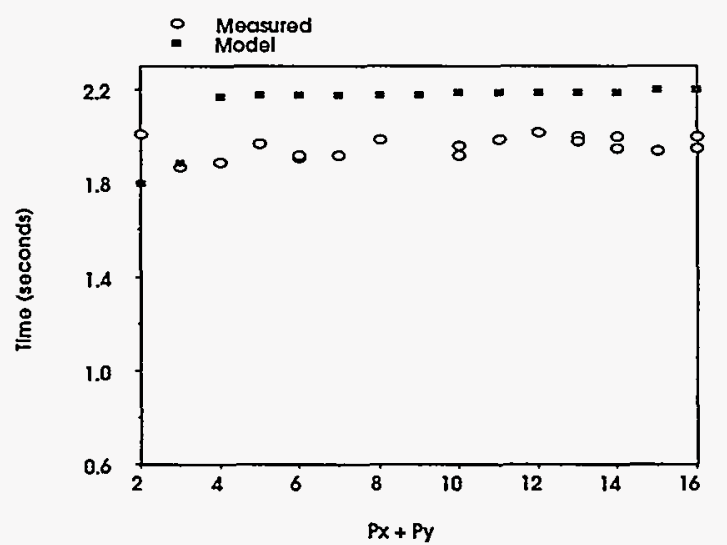

Figure 15. $T^{\text {romp }}$ dominant. $16 \times 16 \times 1000 . N_{\text {sweep }}$ large. IBM RS/6000 SP.

\subsection{Strong Scalability}

In a "strong scalability" analysis, the overall problem size remains constant as the processor configuration increases. Therefore, $T_{\text {msg }}$ and $T_{\text {cpu }}$ vary from run to run as the subgrid size decreases. In Figure 16 the comparison between measured and modeled time for the strong scalability analysis out to nearly 500 processors on the problem size $50 \times 50 \times 50$ is shown. The agreement is excellent.

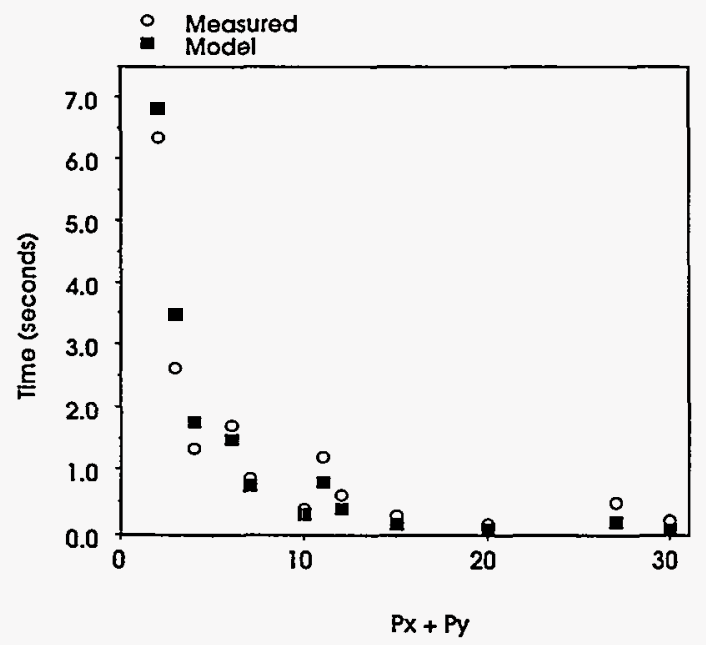

Figure 16. Strong Scalability. CRAY T3E.

\subsection{Blocking tradeoffs}

It is of interest to investigate whether the model captures the variation of the elapsed time with the size of the angle- and $\mathrm{k}$-blocks. In particular, it is important that the model correctly predicts the optimal angle- and k- blocking values for different problem sizes.

Intuitively, larger block sizes lead to increased computation/communication ratio due to fewer communication steps and larger message sizes. For wavefront algo- rithms a tradeoff occurs because smaller blocks, while increasing communication time, could lead to better parallel efficiency as the wavefronts have a more rapid succession over the processor array. For specific subgrid size and machine characteristics, unique optimal values for the blocking parameters result from this tradeoff.

Figure 17 shows modeled and experimental data for a $16 \times 16 \times 1000$ subgrid with $10 \mathrm{k}$-planes per block. Compare this with Figure 18 which shows the same data on this subgrid size but with one k-plane per block. A similar comparison using a $6 \times 6 \times 360$ subgrid is presented in Figures 19 and Figure 14 (above). For a $6 \times 6 \times 360$ subgrid size, 10 planes per block leads to lower elapsed time, whereas for the $16 \times 16 \times 1000$ subgrid, 1 plane per block is optimal.

The explanation is that (on the T3E), for the smaller subgrid $(6 \times 6 \times 360)$, larger $k$-blocks are required in order to increase the computation time and decrease communication. In contrast, the larger grid $(16 \times 16 \times 1000)$ already affords a better computation/communication ratio, so that the lower value for the k-block leads to higher parallel efficiency. In this case, the more wavefronts generated, the better the runtime.

The model resolves the tradeoff, predicting accurate values for the blocking parameters for any grid size and machine characteristics.

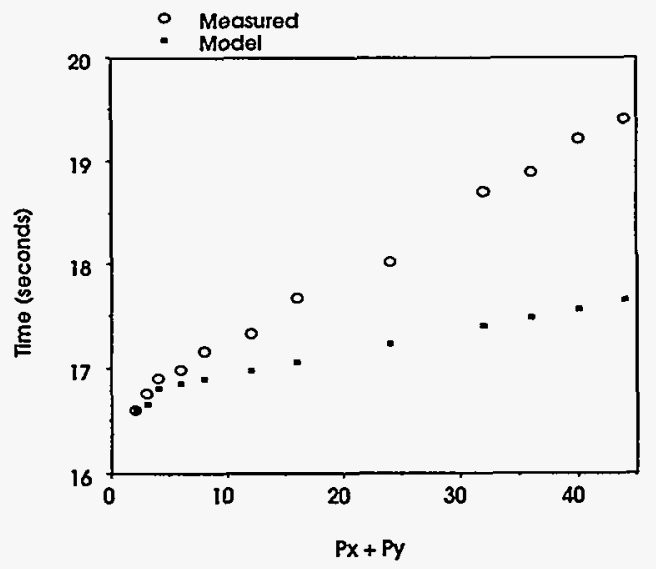

Figure 17. $16 \times 16 \times 1000$. CRAY T3E. $K_{b}=10$. 


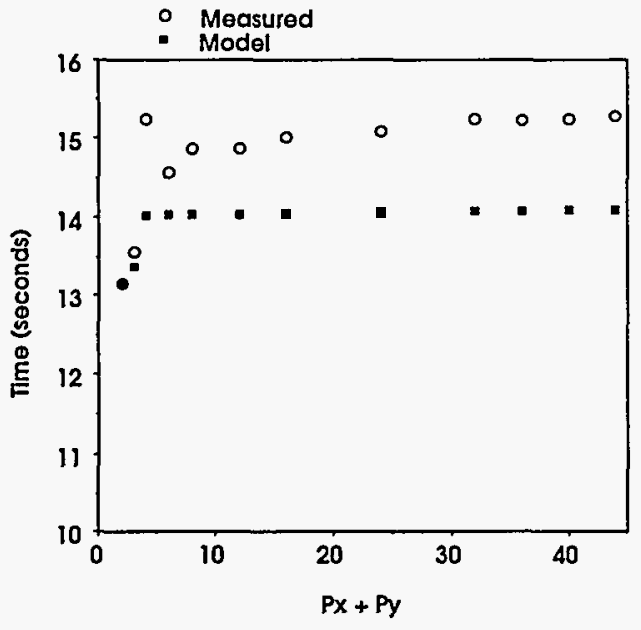

Figure 18. $16 \times 16 \times 1000$. Cray T3E. $K_{b}=1$.

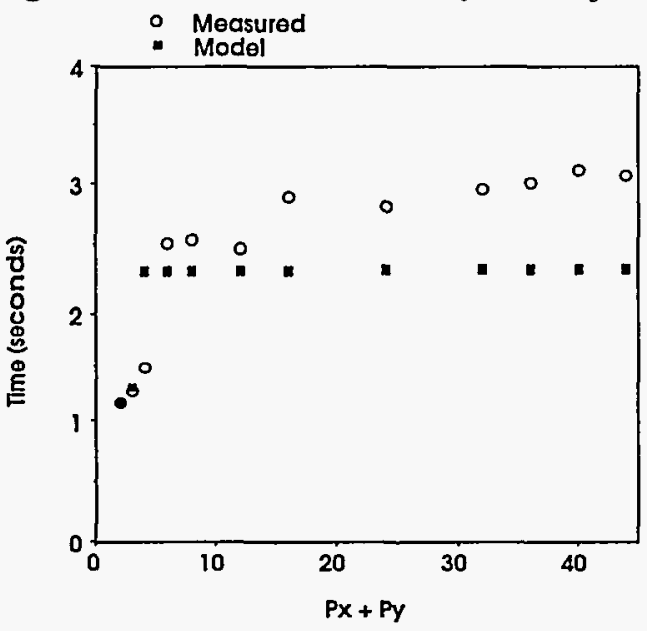

Figure 19. $6 \times 6 \times 360$. CRAY T3E. $K_{b}=1$.

\section{Applications of the Model. Scalability Predictions.}

Performance models of applications are important to computer designers trying to achieve proper balance between performance of different system components. ASCI is targeting a 100-TFLOPS system in the year 2004, with a workload defined by specific engineering needs. In this section we apply our model to predict the machine parameters under which the runtime goal might be met. We assume a 100-TFLOPS-peak system composed of about 20,000 processors (5 GFLOPS peak per processor, an extrapolation of Moore's law).

Three sources of difficulty with such a prognosis are (1) making reasonable estimates of machine performance parameters for future systems; (2) managing the SWEEP3D parameter space (i.e., block sizes); and (3) estimating what problem sizes will be important. We handle the first by studying a range of values covering both conservative and optimistic changes in technology. We handle the second by reporting results that correspond to the shortest execution time (i.e., we use block sizes that minimize runtime). We handle the third as follows.

For particle transport, one ASCI target problem involves $O\left(10^{9}\right)$ mesh points, 30 energy groups, $O\left(10^{4}\right)$ time steps, and a runtime goal of about 30 hours. With 5,000 unknowns per grid point, this requires about 40 TBytes total memory. On 20,000 processors the resulting subgrid size is approximately $6 \times 6 \times 1000$. In a different ASCI scenario, particle transport problem size is determined by external factors. Based on [17], such computations will involve smaller grid sizes ( 20 million cells) but the full resources of the machine are still used. The 20 million-cell problem would utilize a $2 \times 2 \times 250$ subgrid.

\subsection{The 1 billion-cell problem}

Plots showing dependence of runtime with sustained processor speed and latency for MPI communications are shown in Figures 20 and 21 for several k-plane block sizes and using optimal values for the angle-block size. Table 1 collects some of the modeled runtime data for a few important points: Sustained processor speeds of $10 \%$ and $50 \%$ of peak, and MPI latencies of $0.1,1$, and 10 microseconds. Our model shows that the dependence on bandwidth (1/G in LogGP) is small, and as such no sensitivity plot based on ranges for bandwidth is presented. All results assume $400 \mathrm{Mbytes} / \mathrm{s}$ MPI bandwidth [18].

One immediate observation is that runtime under the most optimistic technological estimates in Table 1 is still larger than the 30-hour goal by a factor of two. The execution time goal could be met if, in addition to these values of processor speed and MPI latency (L+o in LogGP), we used what we believe to be an unrealistically high bandwidth value of 4 GBytes/s.

Assuming a more realistic sustained processor speed of $10 \%$ of peak (based on data from today's systems), Table 1 shows that we miss the goal by about a factor of six even when using $0.1 \mu \mathrm{s}$ MPI latency. With the same assumption for processor speed, but with a more conservative value for latency $(1 \mu \mathrm{s})$, the model predicts that we are a factor of 6.6 off. In fact, our results show that the best way to decrease runtime is to achieve better sustained per-processor performance. Changing the sustained processor rate by a factor of five decreases the runtime by a factor of three, while decreasing the MPI latency by a factor of 100 reduces runtime by less than a factor of two. This is a result of the relatively low communication/computation ratio that our model predicts. For example, using values of $1 \mu \mathrm{s}$ and $400 \mathrm{MB} / \mathrm{sec}$ for the communication latency and bandwidth, and a sustained processor speed of 0.5 GFLOPS, the communication time will only be $20 \%$ of the total runtime. 


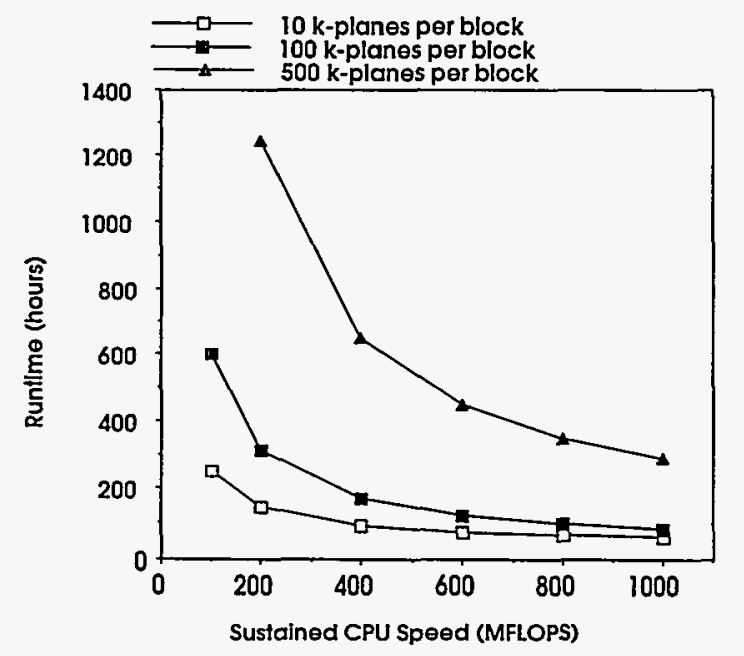

Figure 20. Model-projected sensitivity of the billion-cell transport sweep time to sustained per-processor CPU speed on a hypothetical 100-TFLOPS system for several $\mathrm{k}$-plane block sizes. MPI latency $=15 \mathrm{~ms}$, bandwidth $=$ $400 \mathrm{Mbytes} / \mathrm{s}$.

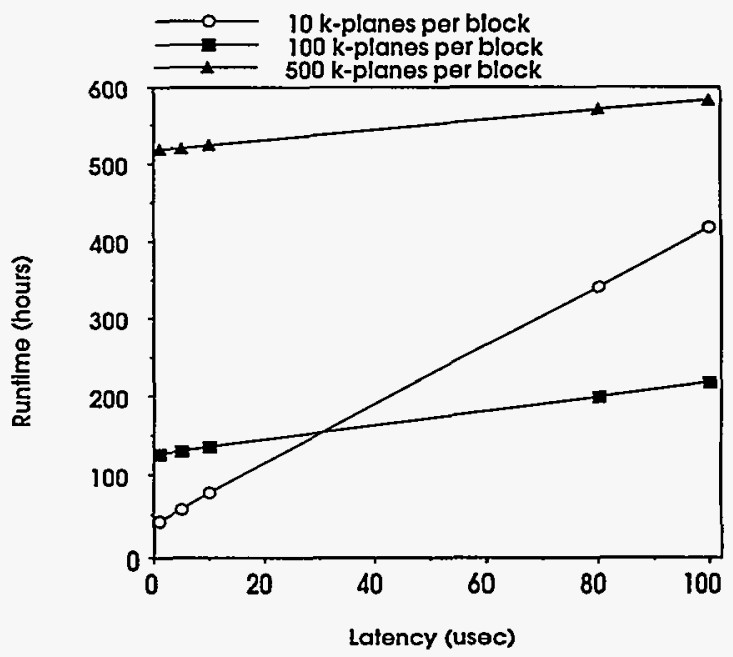

Figure 21. Model-projected sensitivity of the billion-cell transport sweep time to MPI latency on a hypothetical 100-TFLOPS system for several k-plane block sizes. Sustained per-processor CPU speed $=500 \mathrm{MFLOPS}$, bandwidth $=400 \mathrm{Mbytes} / \mathrm{s}$.

\begin{tabular}{|c|c|c|c|c|}
\hline \multirow[b]{2}{*}{$\begin{array}{l}\text { MPI Latency } \\
0.1 \mu \mathrm{s} \\
1.0 \mu \mathrm{s} \\
10 \mu \mathrm{s}\end{array}$} & \multicolumn{2}{|c|}{$10 \%$ of Peak } & \multicolumn{2}{|c|}{$50 \%$ of Peak } \\
\hline & $\begin{array}{c}\text { Runtime (hours) } \\
180 \\
198 \\
291\end{array}$ & $\begin{array}{c}\text { Amount of } \\
\text { Communication } \\
16 \% \\
20 \% \\
20 \%\end{array}$ & $\begin{array}{c}\text { Runtime (hours) } \\
56 \\
74 \\
102\end{array}$ & $\begin{array}{c}\text { Amount of } \\
\text { Communication } \\
52 \% \\
54 \% \\
58 \%\end{array}$ \\
\hline
\end{tabular}

\subsection{The 20 million-cell problem}

Communication is important for this problem size the model predicts that communication time ranges from one-half the total time to two-thirds of the total time depending on specific values for the latency and processor speed. The contribution of the bandwidth to the communication cost is, again, negligible. Figures 22 and 23 show the runtime variation with interprocessor latencies and sustained processor speed, respectively. For this problem size latency and processor speed are equally important in decreasing the runtime, as expected given the fact that the communication time is now a significant component of the total runtime.

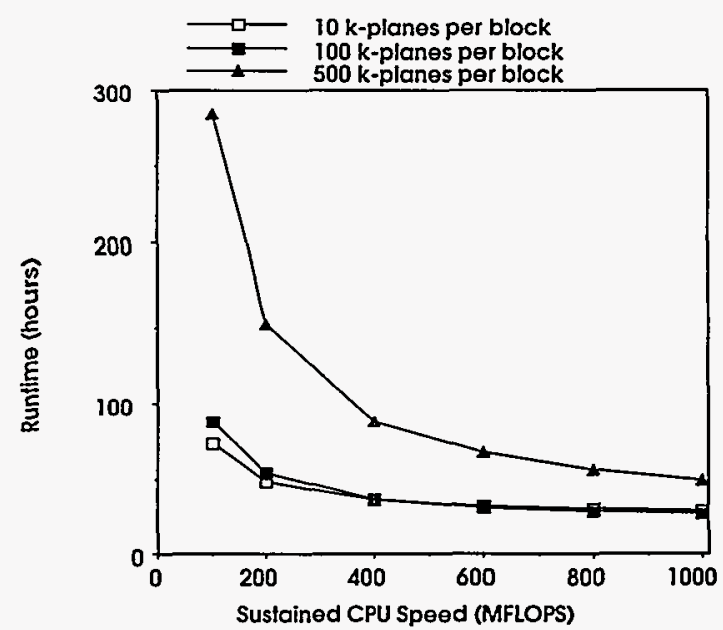

Figure 22. Model-projected sensitivity of the 20 million-cell transport sweep time to sustained perprocessor CPU speed on a hypothetical 100TFLOPS system for several k-plane block sizes. $\mathrm{MPI}$ latency $=15 \mathrm{~ms}$, bandwidth $=400 \mathrm{MB} / \mathrm{s}$. 


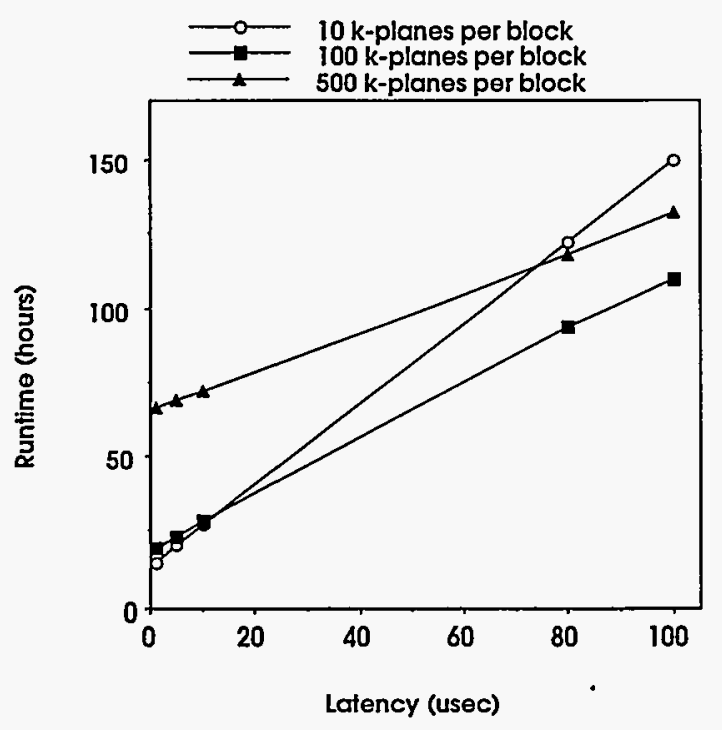

Figure 23. Model-projected sensitivity of the 20 million-cell transport sweep time to MPI latency on a hypothetical 100-TFLOPS system for several $k$ plane block sizes. Sustained per-processor CPU speed $=500 \mathrm{MFLOPS}$, bandwidth $=400 \mathrm{MB} / \mathrm{s}$.

\section{Conclusions}

We introduced a scalability model for parallel, multidimensional, wavefront calculations with machine performance characterized using the LogGP framework. The model accounts for overlap in the communication and computation components. The agreement with experimental data is very good under a variety of model sizes, data partitionings, blocking strategies, and on three different parallel architectures. Using our model, we analyzed performance of a deterministic transport code on a hypothetical future parallel system of interest to ASCI. A proposed 100-TFLOPS system with conservative estimates for communication bandwidth and latency improvements would not be capable of running a billion-point ASCI $S_{N}$ problem within time-limit goals. Our analysis showed that contrary to conventional wisdom, interprocessor communication performance was not the bottleneck for such a problem, although communication does became important for smaller problem sizes. For the largest problem, single-node efficiency was the dominant factor.

\section{Acknowledgements.}

We thank Randal Baker and Ken Koch of LANL Groups X-CM and X-TM for many helpful discussions and for providing several versions of the SWEEP3D benchmark. We thank Vance Faber and Madhav Marathe of LANL Group CIC-3 for interesting discussions regarding mapping problem meshes to processor topologies. We acknowledge the use of computational resources at the Advanced Computing Laboratory, Los Alamos National Laboratory, and support from the U.S. Department of Energy under Contract No. W-7405-ENG-36. We also thank Pat Fay of Intel Corporation for help running SWEEP3D on the Sandia National Laboratory ASCI Red TFLOPS system, and SGI/CRAY for a generous grant of computer time on the CRAY T3E system. We also acknowledge the use of the IBM SP2 at the Lawrence Livermore National Laboratory.

\section{References.}

1. G. F. Pfister, In Search of Clusters - The Coming Battle in Lowly Parallel Computing, Prentice Hall PTR, Upper Saddle River, NJ, 1995, pages 219223.

2. L. Lamport, The Parallel Execution of DO Loops," Communications of the ACM, 17(2):83:93, ?., 19 ?.

3. K. R. Koch, R. S. Baker and R. E. Alcouffe, "Solution of the First-Order Form of the 3-D Discrete Ordinates Equation on a Massively Parallel Processor," Trans. of the Amer. Nuc. Soc., 65, 198, 1992.

4. W. D. Joubert, T. Oppe, R. Janardhan, and W. Dearholt, "Fully Parallel Global M/ILU Preconditioning for 3-D Structured Problems," to be submitted to SIAM J. Sci. Comp.

5. J. Qin and T. Chan, "Performance Analysis in Parallel Triangular Solve," IEEE Second International Conference on Algorithms \& Architectures for Parallel Processing, pp 405-412, June 1996.

6. M. T. Heath and C. H. Romine, "Parallel Solution of Triangular Systems on Distributed Memory Multiprocessors," SIAM J. Sci. Statist. Comput. Vol. 9, No. 3, May 1988.

7. R. F. Van der Wijngaart, S. R. Sarukkai, and P. Mehra, "Analysis and Optimization of Software Pipeline Performance on MIMD Parallel Computers," Technical Report NAS-97-003, NASA Ames Research Center, Moffett Field, CA, February, 1997.

8. R. E. Alcouffe, "Diffusion Acceleration Methods for the Diamond-Difference DiscreteOrdinates Equations," Nucl. Sci. Eng. $\{64\}, 344$ (1977).

9. R. S. Baker and R. E. Alcouffe, "Parallel 3-D $S_{N}$ Performance for DANTSYS/MPI on the CRAY T3D, Proc. of the Joint Intl'1 Conf. On Mathematical Methods and Supercomputing for Nuclear Applications, Vol 1. page 377, 1997.

10. M. R. Dorr and E. M. Salo, "Performance of a Neutron Transport Code with Full Phase Space Decomposition and the CRAY Research T3D," ???

11. R. S. Baker, C. Asano, and D. N. Shirley, "Implementation of the First-Order Form of the 3-D 
Discrete Ordinates Equations on a T3D, Technical Report LA-UR-95-1925, Los Alamos National Laboratory, Los Alamos, NM, 1995; 1995 American Nuclear Society Meeting, San Francisco, CA, 10/2911/2/95.

12. M. R. Dorr and C. H. Still, "Concurrent Source Iteration in the Solution of Three-Dimensional Multigroup Discrete Ordinates Neutron Transport Equations," Technical Report UCRL-JC-116694, Rev 1, Lawrence Livermore National Laboratory, Livermore, CA, May, 1995.

13. E. E. Lewis and W. F. Miller, Computational Methods of Neutron Transport, American Nuclear Society, Inc., LaGrange Park, IL, 1993.

14. R. E. Alcouffe, R. Baker, F. W. Brinkley, Marr, D., R. D. O'Dell and W. Walters, "DANTSYS: A Diffusion Acclerated Neutral Particle Transport Code," Technical Report LA-12969-M, Los Alamos National Laboratory, Los Alamos, NM, 1995.

15. R. S. Baker, K. R. Koch, "An Sn Algorithm for the Massively Parallel CM200 Computer", Nucl. Sci. and Eng., Vol 128, No. 3, p. 312, 1998.

16. D. Culler, R. Karp, D. Patterson, A. Sahay, E. Santos, K. Schauser, R. Subramonian, and T. von Eiken, "LogP: A Practical Model of Parallel Computation," Communications of the ACM, 39(11):79:85, Nov., 1996.

17. R. S. Baker (LANL), Private Communication, June 1998.

18. C. Holt, M. Heinrich, J. P. Singh, E. Rothberg, and J. L. Hennessy, "Effects of Latency, Occupancy, and Bandwidth in DSM Multiprocessors," Stanford Univ. Comp. Sci. Report CSL-TR-95-660, 1/95. 